\title{
Diseño de un módulo experimental bioclimático obtenido a partir del análisis de simulaciones térmicas para el centro poblado de Imata (4519 m s.n.m.) ubicado en Arequipa, Perú
}

\author{
Juan O. Molina(1), Gilles Lefebvre ${ }^{(2)}$, Manfred Horn ${ }^{(1)}$, y Mónica M. Gómez ${ }^{(1) \star}$ \\ (1) Facultad de Ciencias, Universidad Nacional de Ingeniería, Av. Túpac Amaru 210, Rímac, Lima-Perú \\ (correo-e: juan.molina.f@uni.pe; mhorn@uni.edu.pe;mgomez@uni.edu.pe) \\ (2) Centro de Estudio e Investigación en Térmica, Medio Ambiente y Sistemas, Universidad Paris Este, IUT/CERTES, 61 \\ Av. General de Gaulle, 94010 Créteil Francia (correo-e: lefebvre@u-pec.fr) \\ ${ }^{*}$ Autor a quien debe ser dirigida la correspondencia.
}

Recibido Ago. 16, 2019; Aceptado Oct. 11, 2019; Versión final Nov. 30, 2019, Publicado Abr. 2020

\begin{abstract}
Resumen
En base a simulaciones térmicas dinámicas con el software EnergyPlus se propone un módulo experimental de un espacio habitable para el poblado de Imata, Perú, a $4519 \mathrm{msnm}$, con condiciones climáticas severas de muy bajas temperaturas. Se realizó simulaciones para cinco configuraciones del módulo, añadiendo sucesivamente componentes bioclimáticas, tales como mejoras en el aislamiento de la envolvente, tragaluz e invernadero adosado. Para el mes de más bajas temperaturas, la temperatura media en el módulo de mejor configuración es $11^{\circ} \mathrm{C}$ superior a la temperatura media en el módulo correspondiente a espacios habitables típicos de Imata. La temperatura nocturna promedia mínima es $18.4^{\circ} \mathrm{C}$ superior a la correspondiente temperatura exterior $\left(-4^{\circ} \mathrm{C}\right.$ ). Para mantener en el interior una temperatura de $16.3^{\circ} \mathrm{C}$ (modelo adaptivo), se requiere, en promedio anual, una energía diaria de $12 \mathrm{kWh}$ para el módulo típico y $1 \mathrm{kWh}$ para el mejor diseño.
\end{abstract}

\section{Design of an experimental bioclimatic module obtained from the analysis of thermal simulations for the community of Imata (4519 m a.s.I.), located in Arequipa, Perú}

\begin{abstract}
Based on dynamic thermal simulations with the EnergyPlus software, an experimental module of a rural dwelling is proposed for the village of Imata, Peru, at 4519 meters above sea level, with severe climatic conditions of very low temperatures. Simulations were carried out for five module configurations, successively adding bioclimatic components, such as improvements in the insulation of the enclosure, skylight and attached greenhouse. For the month with the lowest temperatures, the average temperature in the best configuration module is $11^{\circ} \mathrm{C}$ higher than the average temperature in the module corresponding to typical Imata houses. The minimum average night temperature is $18.4^{\circ} \mathrm{C}$ higher than the corresponding outside temperature $(-4$ ${ }^{\circ} \mathrm{C}$ ). To maintain a temperature of $16.3^{\circ} \mathrm{C}$ inside the module (adaptive model), on annual average, a daily energy of $12 \mathrm{kWh}$ is required for the typical module, and $1 \mathrm{kWh}$ for the best design.
\end{abstract}




\section{INTRODUCCIÓN}

La envolvente de una vivienda, en especial las propiedades termo-físicas de los materiales que la conforman, van a proporcionar, en mayor o menor grado, el bienestar térmico y la eficiencia energética dentro de la vivienda (se usará la expresión "bienestar térmico" en vez del usual "confort térmico", dado que el término "confort" posee una connotación de comodidad que no se ajusta al contexto del presente trabajo). También resulta importante considerar la inercia térmica de los materiales en el interior de la vivienda. En conjunto estos parámetros van a crear ambientes saludables térmicamente con el consecuente ahorro energético. Se desconoce, o por lo menos, no es prioritaria esta consideración en las poblaciones rurales Altoandinas del Perú, dado a que cada vez más se masifica el uso de materiales incompatibles con el clima, como por ejemplo las planchas de acero galvanizado onduladas (denominadas "calamina metálica", en el Perú), un material económico y fácil de instalar pero que no brinda protección alguna frente a la severidad del frío extremo que se alcanza durante las "heladas" (nombre con el que se conoce a fenómenos climatológicos cuando las temperaturas descienden por debajo de $0^{\circ} \mathrm{C}$ ), y que se presentan en los meses de invierno con mayor intensidad en la sierra sur del Perú (Figura 1). Específicamente en el Centro Poblado de Imata (Altura: 4519 msnm, Latitud: $15^{\circ} 55^{\prime} 43^{\prime \prime} \mathrm{S}$ y Longitud: $\left.71^{\circ} 06^{\prime} 31^{\prime \prime} \mathrm{O}\right)$, se han registrado temperaturas de hasta $-23^{\circ} \mathrm{C}$ (SENAMHI-FAO, 2010). Estas condiciones climáticas, al sobrepasar los umbrales de adaptación de la población, ocasionan daños a la vida, salud, educación, actividades productivas (agrícolas y ganaderas) e infraestructura. Siendo afectadas principalmente las poblaciones que se encuentran en situación de alta vulnerabilidad debido a su condición social (pobreza y pobreza extrema) y su desarrollo biológico (niños, adultos mayores y personas con habilidades especiales). Según el Instituto Nacional de Defensa Civil (INDECI), la vulnerabilidad a las bajas temperaturas de la población asentada a más de $3000 \mathrm{msnm}$, se debe entre otras cosas, a las viviendas inadecuadas con las que cuenta la población, y al desconocimiento de conceptos de ventilación y aprovechamiento de la energía solar.

Paradójicamente, frente a la realidad anterior, en las zonas rurales Altoandinas al sur del Perú, como el Centro Poblado de Imata, se reciben altos grados de radiación solar. Así, el promedio diario durante un año de la densidad superficial de energía solar se encuentra entre 5 a $6\left(\mathrm{kWh} / \mathrm{m}^{2}\right.$ día) (Gómez et al., 2016), como se puede observar en el mapa elaborado por el Banco Mundial (Figura 2). Entonces es un reto nacional aprender a captar, almacenar y distribuir esta energía al interior de la vivienda para alcanzar un grado de bienestar térmico que brinde un ambiente saludable a la población, lo que además repercutirá en una reducción de la demanda de energía para calefacción, en este caso, la reducción del uso de leña.

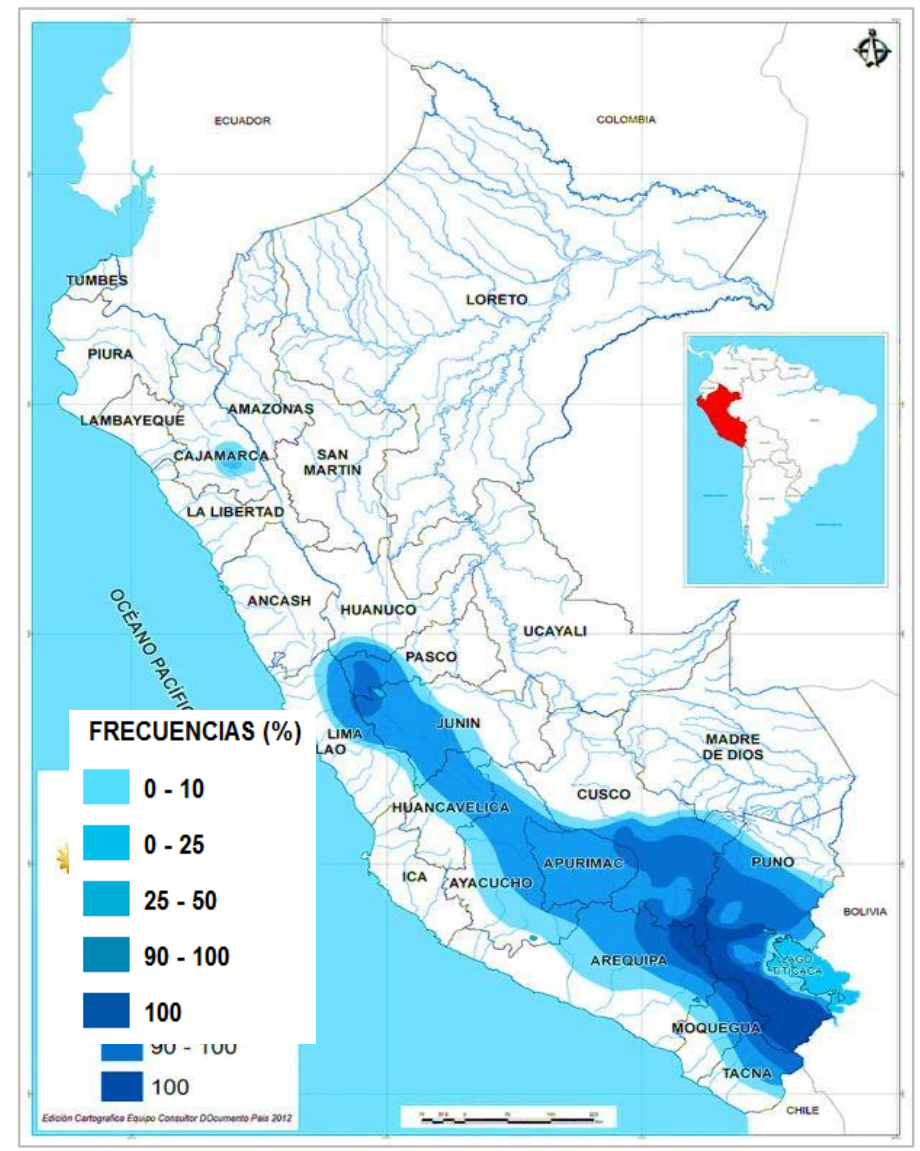

Fig.1: Mapa del Perú de frecuencias de "heladas" para el mes de julio del año 2000 (PREDES, 2016). 


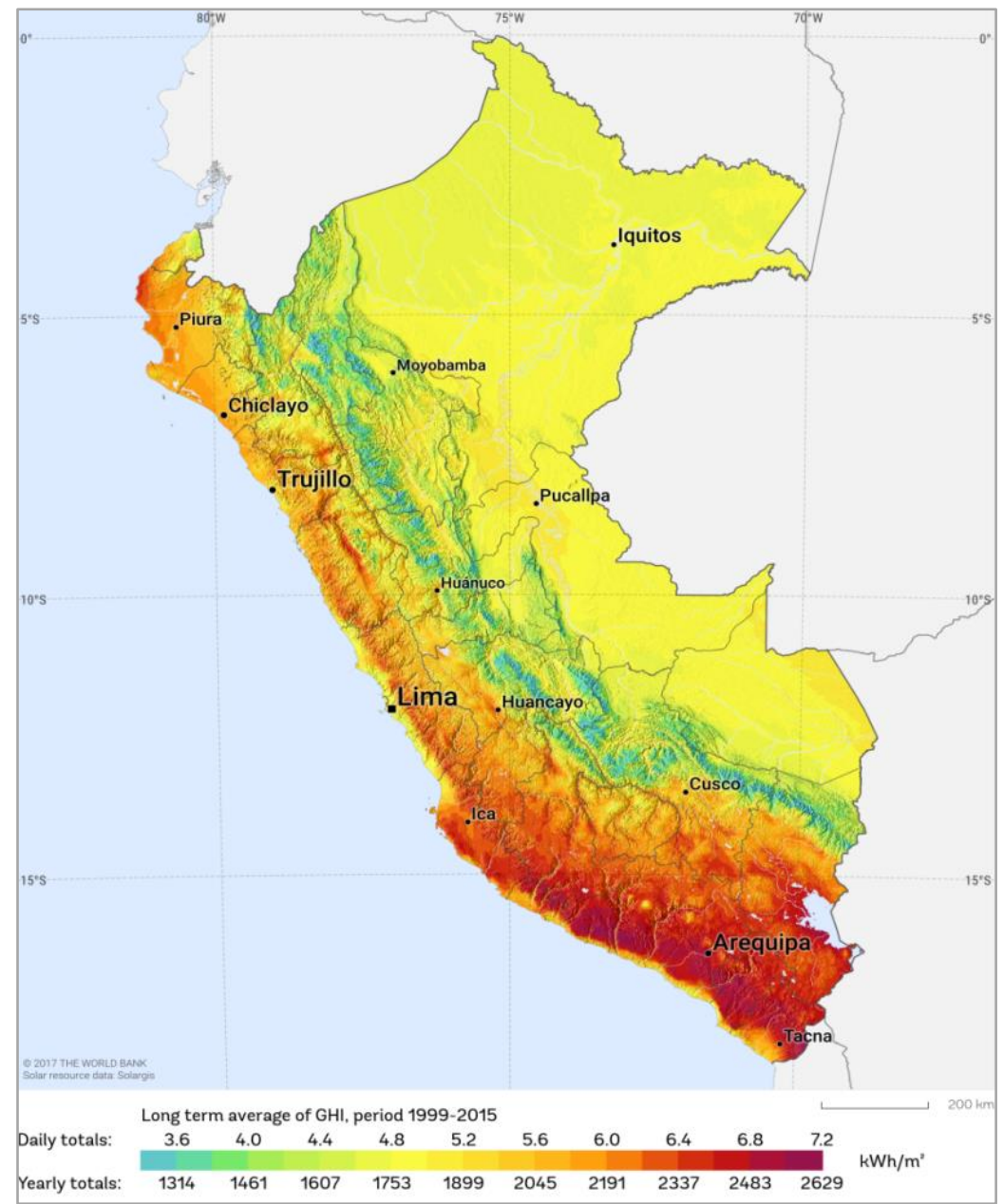

Fig. 2: Mapa del Perú de Irradiación de energía solar promedio diaria anual (World Bank Group, 2019).

La disponibilidad de la energía solar en Imata, estimada según datos del archivo de clima para un año meteorológico típico generado con el Programa Meteonorm (Tsoka et al., 2017), es abundante (Figura 3), y se destaca que en la mayor parte del año el Sol se encuentra al norte (Figura 4) y, para las fechas del 2 de noviembre y 7 febrero, se encontrará a medio día en la posición del cenit. Durante ocho meses el promedio de la irradiación diaria de energía solar, que incide sobre una superficie horizontal, supera los $6\left(\mathrm{kWh} / \mathrm{m}^{2}\right.$ día), e incluso, durante el mes de noviembre, sobrepasa los $8\left(\mathrm{kWh} / \mathrm{m}^{2}\right.$ día).

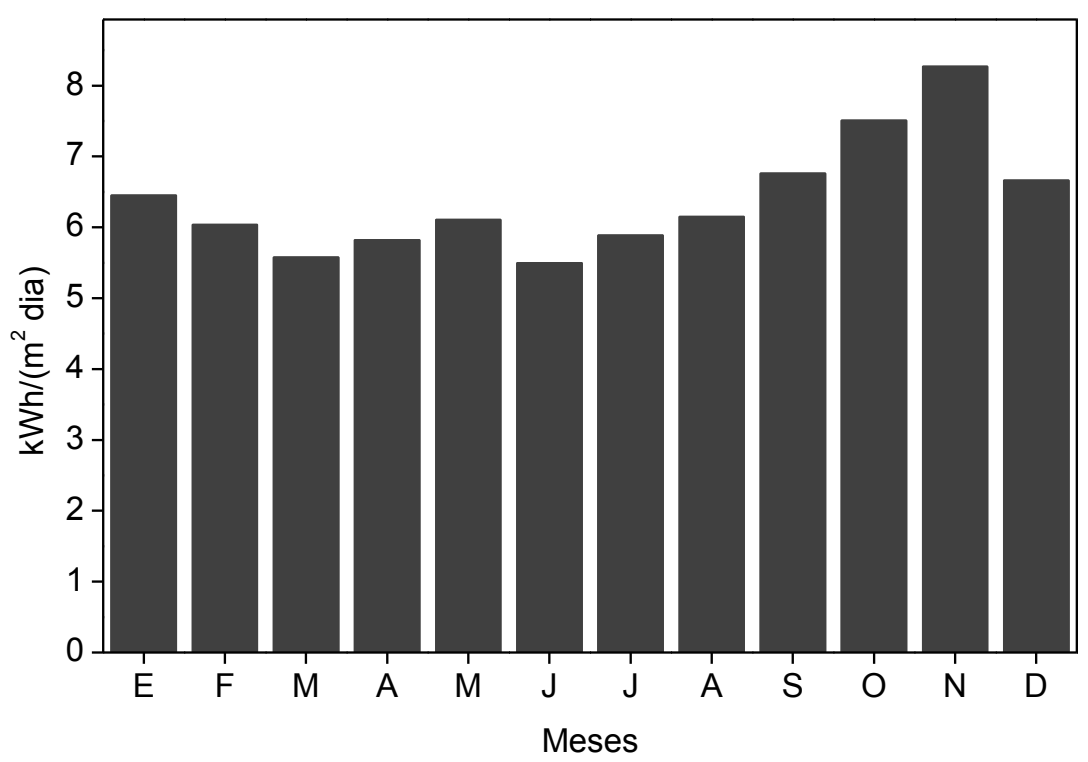

Fig. 3: Energía solar media diaria ( $\mathrm{kWh} / \mathrm{m}^{2}$ día) por mes para Imata según archivo de clima generado con el Programa Meteonorm. 


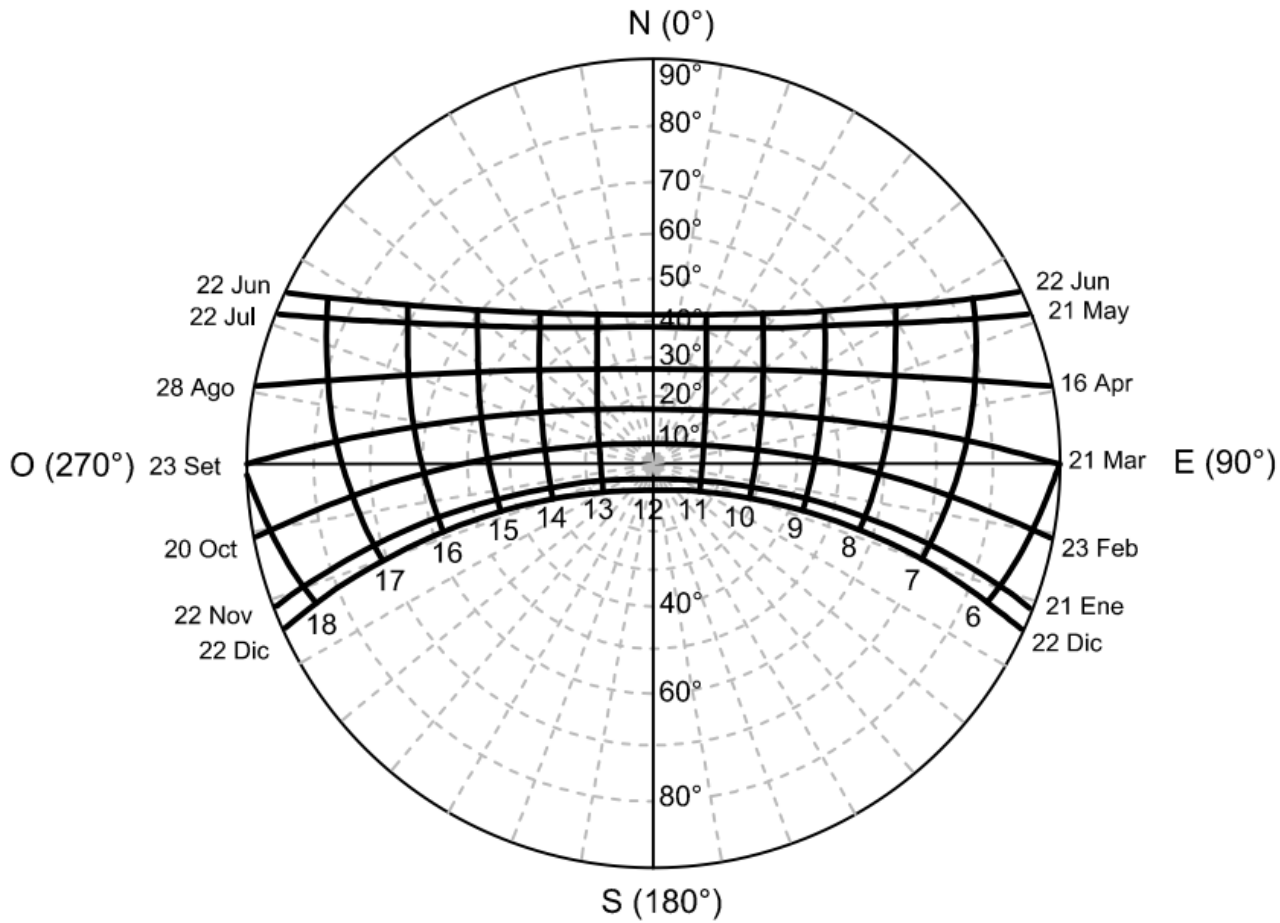

Fig. 4: Carta solar estereográfica para el Centro Poblado de Imata (4519

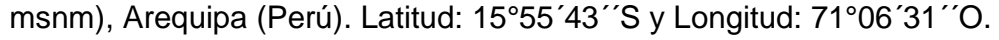

En estudios de latitudes diferentes del Perú se considera la zona de bienestar térmico en un rango de 21 a $26{ }^{\circ} \mathrm{C}$ (zona 1 en la Figura 5) (ASHRAE, 1992; Givoni, 1992). Sin embargo, utilizando un enfoque adaptativo (Nicol y Humphreys, 2002), la zona de bienestar térmico en una vivienda depende de la temperatura exterior $y$, para calcularla, es necesario conocer la temperatura promedio mensual en el exterior (cuyo rango se representa en líneas rectas a colores en la Figura 5). Además, para diferencias de temperatura del aire entre el día y la noche inferiores a $13^{\circ} \mathrm{C}$, se recomienda una oscilación de la amplitud de $\pm 2.5^{\circ} \mathrm{C}$ (Morillón, 2004), lo que resulta en una temperatura de bienestar térmico mensual para Imata de $16.3^{\circ} \mathrm{C}$. Esto indicaría que el bienestar térmico en una vivienda ubicada en climas fríos, como en Imata, podría alcanzarse básicamente por estrategias bioclimáticas de calentamiento solar pasivo. En la Figura 6, los rangos de las temperaturas para el bienestar térmico se presentan entre las líneas negras punteadas adaptadas del Programa Meteonorm.

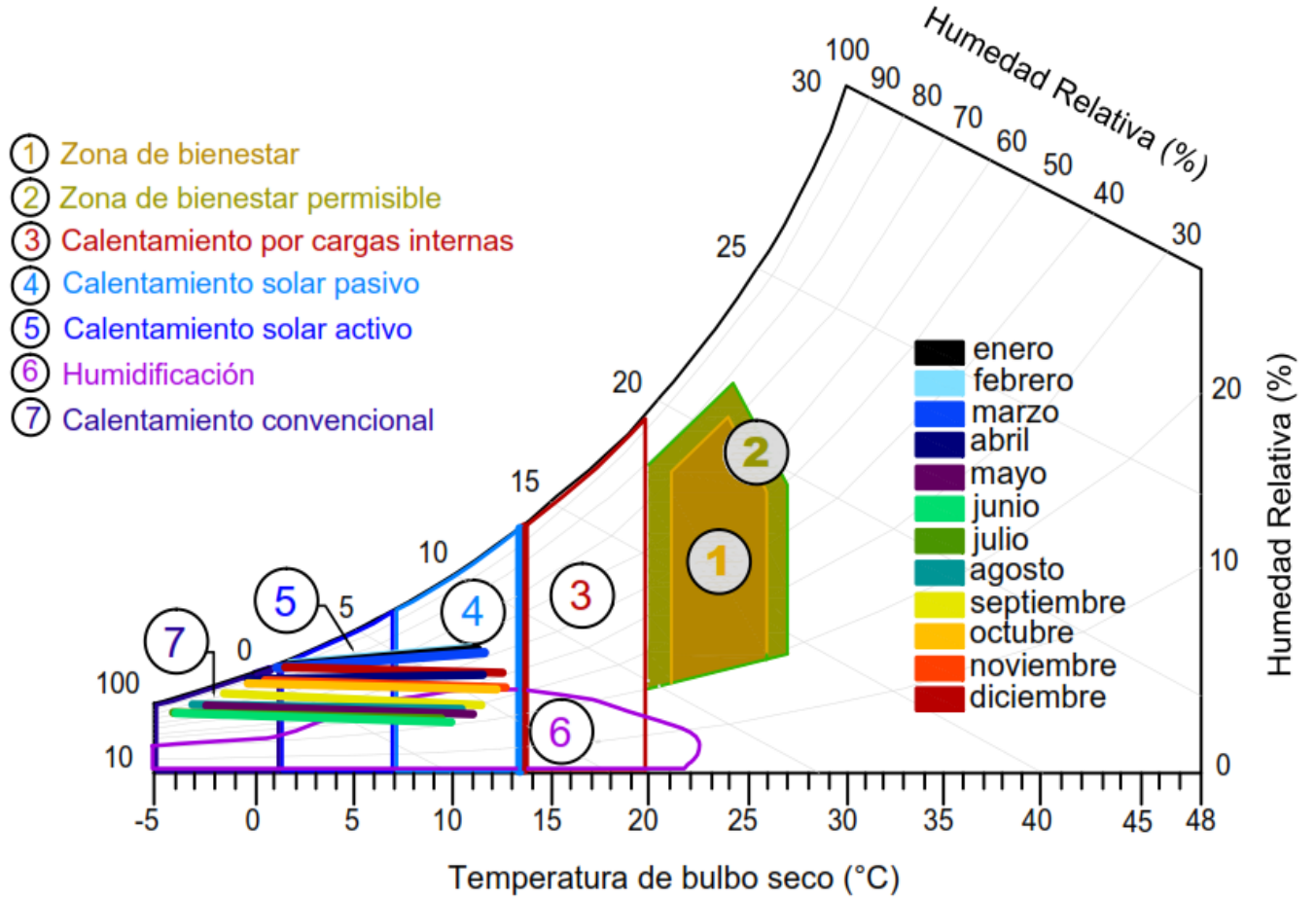

Fig. 5: Cuadro bioclimático que resume las estrategias de diseño para un clima frío. (Adaptado de Agugliaro et al., 2015). 


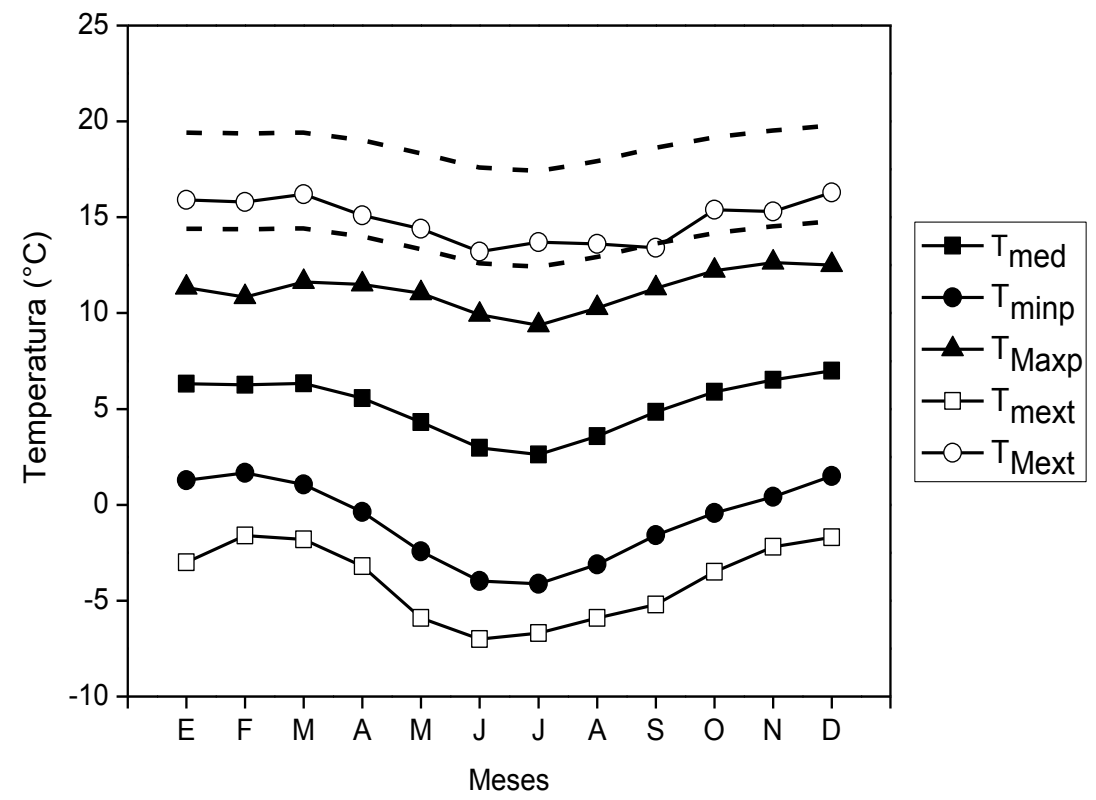

Fig. 6: Temperaturas promedio mínimas ( $\left.T_{\operatorname{minp}}\right)$ y máximas ( $\left.T_{M a x p}\right)$ diarias mensuales, temperaturas extremas mínimas ( $T_{\text {mext }}$ y máximas ( $T_{\text {Mext }}$ ) diarias mensuales para un año meteorológico típico de Imata.

Asimismo, el uso de materiales tradicionales de tierra de alta masa térmica como el adobe (Abanto et al., 2017) o tapial (Velazco et al., 2017), va a permitir colectar y almacenar el calor del Sol en el día dentro de su masa, siendo capaz de desplazar el tiempo y aplanar las fluctuaciones de flujo de calor (Verbeke y Audenaert, 2018). Esto se conoce como inercia térmica que es la propensión de un material a mantener durante un tiempo prolongado su temperatura original cuando interviene una perturbación de su equilibrio térmico. Si la perturbación lo lleva a una nueva temperatura de equilibrio, esta inercia térmica representa la "lentitud" con que se alcanza este nuevo punto de equilibrio (Lefebvre, 1989).

Debido a los complejos cálculos numéricos que se requieren, es difícil determinar el rendimiento térmico y energético de una edificación en condiciones transitorias reales donde el material puede absorber, almacenar y liberar progresivamente el calor. Por lo tanto, muchas normas de países Sudamericanos que se refieren al bienestar (confort) térmico con eficiencia energética (Silvero et al., 2019), incluida la Norma Técnica Peruana EM.110 "Confort Térmico y Lumínico con Eficiencia Energética" (MVCS, 2014), basan su análisis de la transferencia de calor en un estado estacionario, donde solo se tiene que estimar la resistencia térmica, o su inversa (transmitancia térmica), de los elementos constructivos como un indicador de su rendimiento térmico y nivel de aislamiento, ignorando su capacidad para almacenar calor.

Sin embargo, ahora hay herramientas numéricas computacionales, como los programas dinámicos de simulación térmica/energética, que durante las últimas décadas se han convertido en una característica estándar en el diseño de edificios en todo el mundo (Harish y Kumar, 2016). Algunos de estos programas son de código abierto y uso gratuito como EnergyPlus (Crawley et al., 2001), que realiza simulaciones de edificios y cuya autoría pertenece al Departamento de Energía de los Estados Unidos, una herramienta generalizada y aceptada en la comunidad científica que realiza análisis de energía de edificio en todo el mundo (Fumo et al., 2010). EnergyPlus es compatible con los programas OpenStudio y SketchUp, que van a permitir realizar diseños en 3D, ambos softwares también son de código abierto y de uso gratuito (Alghoul et al., 2017). Otro programa de uso libre es el DOE-2.1, utilizado para cálculos de consumos energéticos como el estudio desarrollado por (Calderón et al., 2011) que compara dos viviendas denominadas eficiente e ineficiente y cuya simulación, arroja consumos altos de energía para la vivienda ineficiente que puede reducirse entre 40 a 50 $\%$, si la vivienda es eficiente. O otros ejemplos se tienen con los programas comerciales Trnsys y Accurate, con el primero se estimó el comportamiento de la temperatura interior y demanda de energía de un local comercial (Madrigal et al., 2018), y con el segundo se realizó el análisis térmico de edificios residenciales y cálculo de demanda de energía (Albatayneh et al., 2017).

En el Perú, se han llevado a cabo pocos estudios aplicando la simulación dinámica térmica/energética en la etapa de diseño de una edificación, entre los que destacan: El estudio realizado en Raymina (Ayacucho) sobre el modelado térmico de un módulo experimental de vivienda Altoandina, que utiliza los programas OpenStudio y EnergyPlus, para determinar su rendimiento térmico (Molina et al., 2019). Y el estudio llevado a cabo en llave (Puno), donde se utilizó el programa EnergyPlus para comparar los resultados con las medidas experimentales en una vivienda solar (Flores, 2014). 


\section{METODOLOGÍA}

La metodología del presente estudio comprende realizar un análisis del rendimiento térmico/energético de cinco variantes de un módulo experimental de vivienda (ME) de $3 \mathrm{~m} \times 4 \mathrm{~m}$, en base al programa EnergyPlus, y sus complementos SketchUp, y OpenStudio. Posteriormente se pretende construir dos de estos módulos en una zona Altoandina del Perú. La simulación se inicia con el modelado en SketchUp que es un programa de diseño gráfico en 3D y en cuyo entorno se integran las herramientas del OpenStudio con el cual se crean principalmente las zonas térmicas (volúmenes de aire a temperatura uniformes), y archivo IDF de entrada al EnergyPlus que contiene la geometría del edificio. La simulación se realiza con el EnergyPlus que es el motor de cálculo que utiliza balances energéticos de transferencia de calor dependientes del tiempo, para determinar la temperatura interior, y la demanda de energía de calefacción y/o refrigeración en un edificio, su ejecución depende entre otras cosas, de dos archivos de ingreso importantes, el IDF que contiene la geometría del edificio, y el archivo de clima EPW principalmente de un año típico meteorológico.

\section{Características del Módulo Experimental (ME)}

EI ME estará ubicado en el centro poblado de Imata, distrito de San Antonio de Chuca, provincia de Caylloma, región de Arequipa (Perú), ubicado en una zona Altoandina con una latitud de $15^{\circ} 55^{\prime} 43^{\prime \prime}$ Sur, longitud de $71^{\circ} 06^{\prime} 31^{\prime \prime}$ Oeste, y altitud de 4519 metros sobre el nivel del mar. El clima de Imata es de Tundra, categoría ET en la clasificación climática de Köppen-Geiger (Peel et al., 2007). Según el Instituto Nacional de Estadística e Informática (INEI), el 2016 el distrito de San Antonio de Chuca tenía una población de aproximadamente 1500 personas, y la población con alto riesgo de "heladas" era de $33.5 \%$. En la Figura 7a se muestra una vista del centro poblado de Imata donde se aprecia el uso masivo de las planchas de acero galvanizado ondulado como cubierta de techo, principalmente por su bajo coste económico, accesibilidad en el mercado local y practicidad en su instalación. En la imagen también se aprecia una estación meteorológica marca Davis Pro Plus instalada en el cuarto nivel del municipio de Imata (perteneciente a la Universidad Nacional de Ingeniería) para en adelante, contar con data meteorológica real del clima en estudio. La Figura $7 \mathrm{~b}$ muestra parte de la nieve en época de "helada", y la Figura 7c, una vivienda de construcción actual.

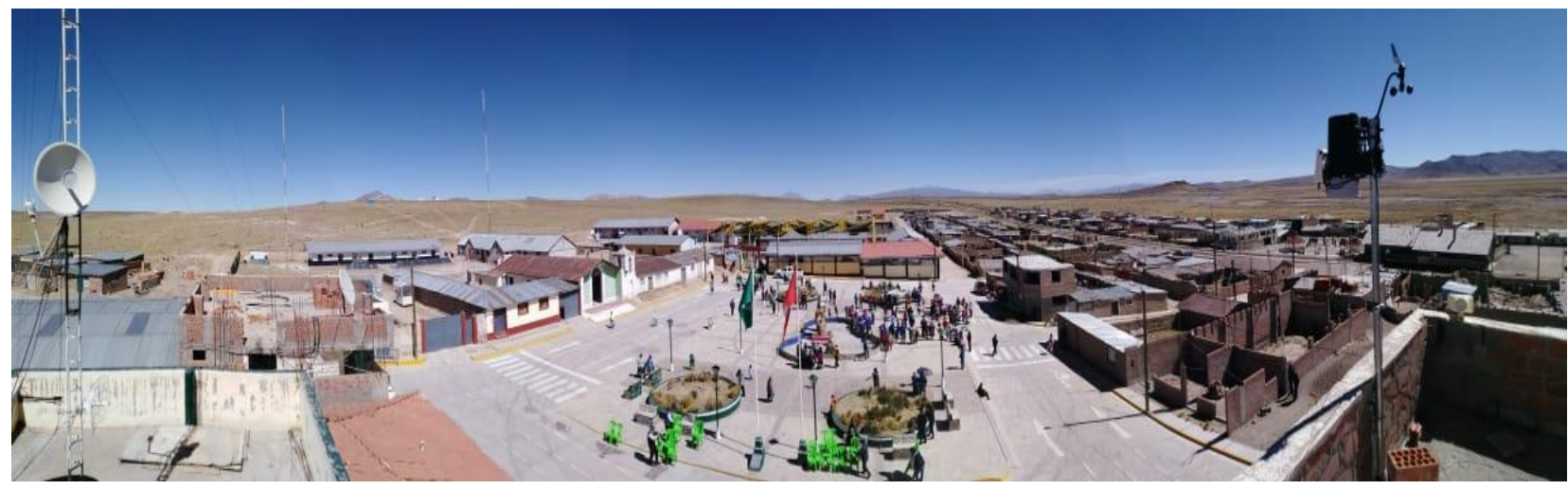

(a)

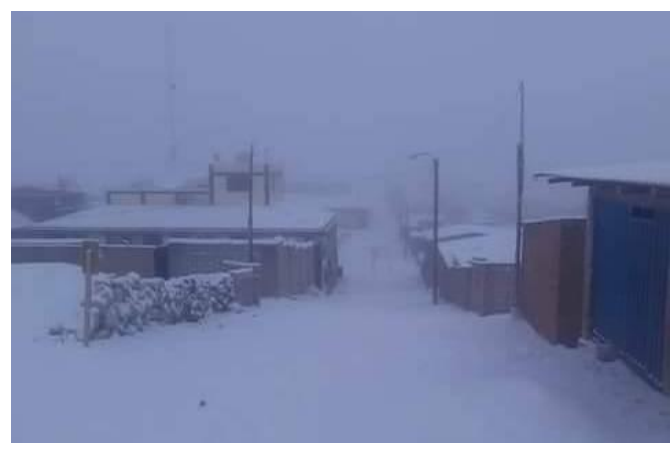

(b)

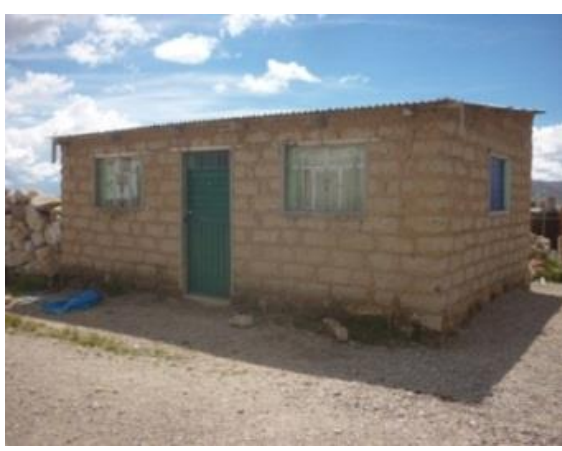

(c)

Fig. 7: Vistas del centro poblado de Imata, (a) panorámica, (b) un día en épocas de "heladas", y (c) vivienda de construcción actual.

La simulación se realizó para el mes de julio donde los valores promedio mensuales de las temperaturas son: media de $2.6^{\circ} \mathrm{C}$, mínimo de $-4.1^{\circ} \mathrm{C}$, y máximo de $9.4^{\circ} \mathrm{C}$, con valores extremos mínimo de $-6.7^{\circ} \mathrm{C}$, y máximo de $16.3^{\circ} \mathrm{C}$ (diciembre). Para la simulación se consideraron tres zonas térmicas del ME: el interior (sala principal), el invernadero adosado, y el ático. Las propiedades termofísicas de los materiales de construcción 
se han tomado de la Norma Técnica Peruana EM.110 "Confort Térmico y Lumínico con Eficiencia Energética," cuyas propiedades térmofísicas de los materiales empleados se muestran en la Tabla 1, el espesor (e), conductividad térmica $(k)$, densidad $(\rho)$, calor específico $(c)$, y resistencia térmica $(R)$. Las propiedades ópticas fueron las recomendadas por el programa EnergyPlus, 0.7 para la absortancia solar, y 0.9 para la absortancia térmica.

Se simularon 5 conformaciones del ME las cuales tuvieron la consideración de mejoras, básicamente, secuenciales, de aislamiento o captación pasiva de energía solar. Así ME1 (Figura 8a) representa la vivienda típica de Imata, es decir, paredes de adobe ( $0.4 \mathrm{~m}$ de espesor), techo y puerta $\left(2 \mathrm{~m}^{2}\right)$ de planchas de acero galvanizado onduladas, piso de tierra apisonada, y tres ventanas (de un solo vidrio con marco metálico), dos de $1 \mathrm{~m}^{2}$ cada una ubicada al este y oeste, y una de $0.8 \mathrm{~m}^{2}$ al norte. Como escala se ha representado a una habitante de $1.6 \mathrm{~m}$ de alto.

Para ME2 (presentado en vista transparente sería lo mismo que la Figura 8a) en relación a ME1 presenta un mejor aislamiento de las paredes por medio de capas de yeso (0.02 $\mathrm{m}$ de espesor) al interior y exterior; el piso se diseña tipo laminar formado por tierra, una manta de plástico, un espacio de aire con marco de madera, y un panel tipo sándwich (AIPoIAI) cuyo centro es de poliuretano expandido de $0.04 \mathrm{~m}$ de espesor, y extremos de chapa de aluzinc de $0.5 \mathrm{~mm}$ para dar rigidez al poliuretano; las ventanas se consideran con doble vidrio y marcos de madera, cada una lleva contraventana a $0.1 \mathrm{~m}$ de la superficie interior del vidrio; y la puerta se considera del tipo contraplacada de tableros de triplay separadas una de otra por un espacio de aire de 0.032 m. Para ME3 (Figura 8b), en relación a ME2, se le ha agregado dos tragaluces a ambos lados del techo (al norte y al sur) de $1.2 \mathrm{~m}^{2}$ cada uno, y un falso techo con $2.2 \mathrm{~m}^{2}$ de tragaluz centrado y por debajo de los dos anteriores. La Figura 8c representa una vista de planta de ME1. Para el caso de ME4 (Figura 8d), en relación a ME2, se ha agregado falso techo y se ha adosado un invernadero en la pared norte de un área de $8 \mathrm{~m}^{2}$. Finalmente, ME5 (Figura 8e), en relación a ME4, se ha agregado el tragaluz, como el que se diseña para ME3 pero lleva contraventanas. La construcción en la Figura 8e (ME5) incorpora aislamiento y captación solar pasiva: invernadero adosado y tragaluces. La Figura $8 \mathrm{f}$ es una representación gráfica de vista de planta de ME5.

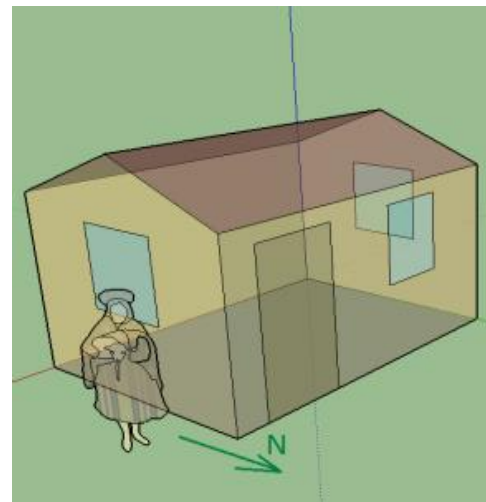

(a)

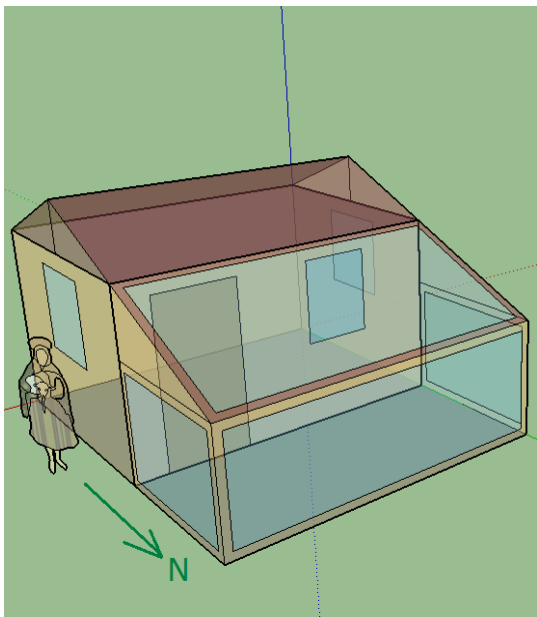

(d)

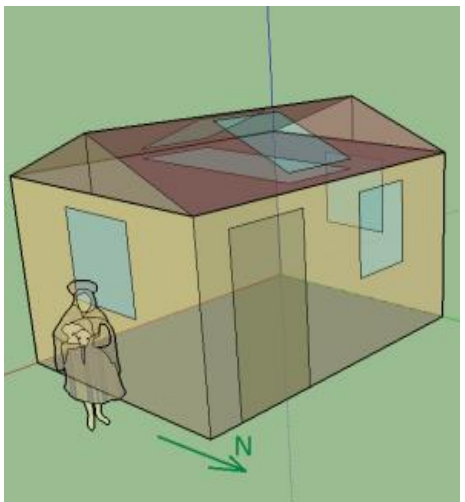

(b)

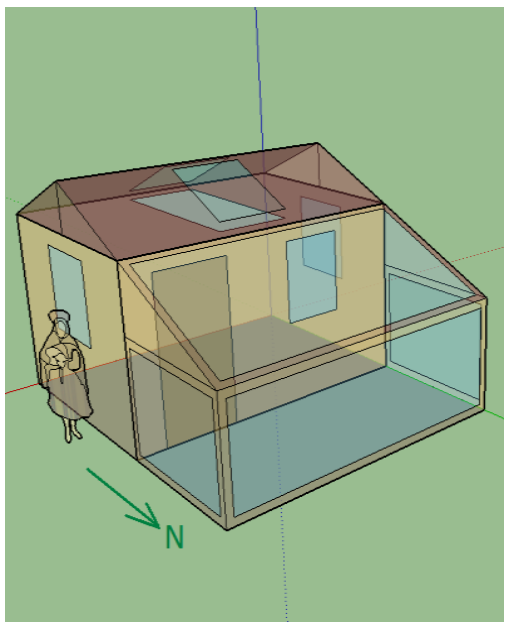

(e)

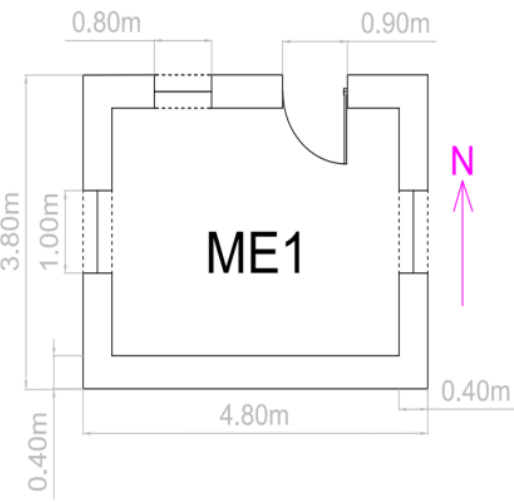

(c)

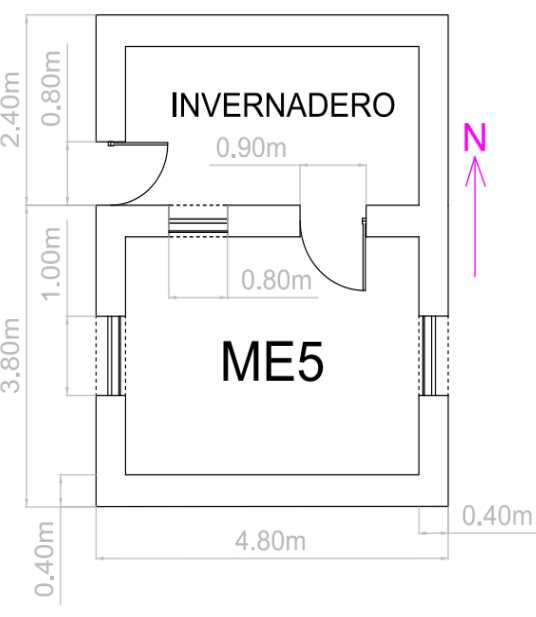

(f)

Fig. 8: Vistas translúcidas de los ME y de planta, (a) ME1, (b) ME3, (c) planta del ME1, (d) ME4, (e) ME5, y (f) planta del ME5. 
Tabla 1: Propiedades termo-físicas de los materiales empleados en la envolvente de los ME (MVCS, 2014).

\begin{tabular}{|l|c|c|c|c|c|}
\hline Material & $\mathrm{e}(\mathrm{m})$ & $\begin{array}{c}\mathrm{k} \\
(\mathrm{W} / \mathrm{m} \mathrm{K})\end{array}$ & $\begin{array}{c}\rho \\
\left(\mathrm{kg} / \mathrm{m}^{3}\right)\end{array}$ & $\begin{array}{c}\mathrm{c} \\
(\mathrm{J} / \mathrm{kg} \mathrm{K})\end{array}$ & $\begin{array}{c}\mathrm{R} \\
\left(\mathrm{m}^{2} \mathrm{~K} / \mathrm{W}\right)\end{array}$ \\
\hline Adobe & 0.40 & 0.95 & 1600 & 920 & 0.42 \\
\hline Tierra & 0.20 & 0.52 & 2050 & 1840 & 0.38 \\
\hline Calamina & 0.0002 & 51.64 & 7838 & 500 & $8.44 \times 10^{-7}$ \\
\hline Yeso & 0.02 & 0.40 & 900 & 1000 & 0.05 \\
\hline Plástico & 0.001 & 0.33 & 925 & 2300 & 0.003 \\
\hline Aluzinc & 0.0005 & 125.50 & 7850 & 523 & $3.98 \times 10^{-6}$ \\
\hline Poliuretano & 0.04 & 0.02 & 40 & 1590 & 1.21 \\
\hline Madera & 0.02 & 0.12 & 510 & 1380 & 0.17 \\
\hline Triplay & 0.004 & 0.14 & 560 & 1400 & 0.029 \\
\hline
\end{tabular}

\section{RESULTADOS Y DISCUSIÓN}

En la Tabla 2 se muestra los resultados de la ejecución de las simulaciones para el mes de julio de las diferentes configuraciones de ME propuestos, evaluando dos rangos de tiempo durante el día: de 18:00 a 8:00 h, y de 8:00 a 18:00 h. Los resultados son los valores de la temperatura máxima diaria promedio mensual, exterior ( $T_{\text {ext }} \mathrm{Mp}$ ) e interior ( $\mathrm{T}_{\text {int }} \mathrm{Mp}$ ), y la temperatura mínima diaria promedio mensual, exterior $\left(\mathrm{T}_{\text {ext }} \mathrm{mp}\right.$ ) e interior (Tint $\mathrm{mp})$.

Tabla 2: Módulos experimentales (ME) simulados con diferentes detalles de elementos constructivos y materiales utilizados.

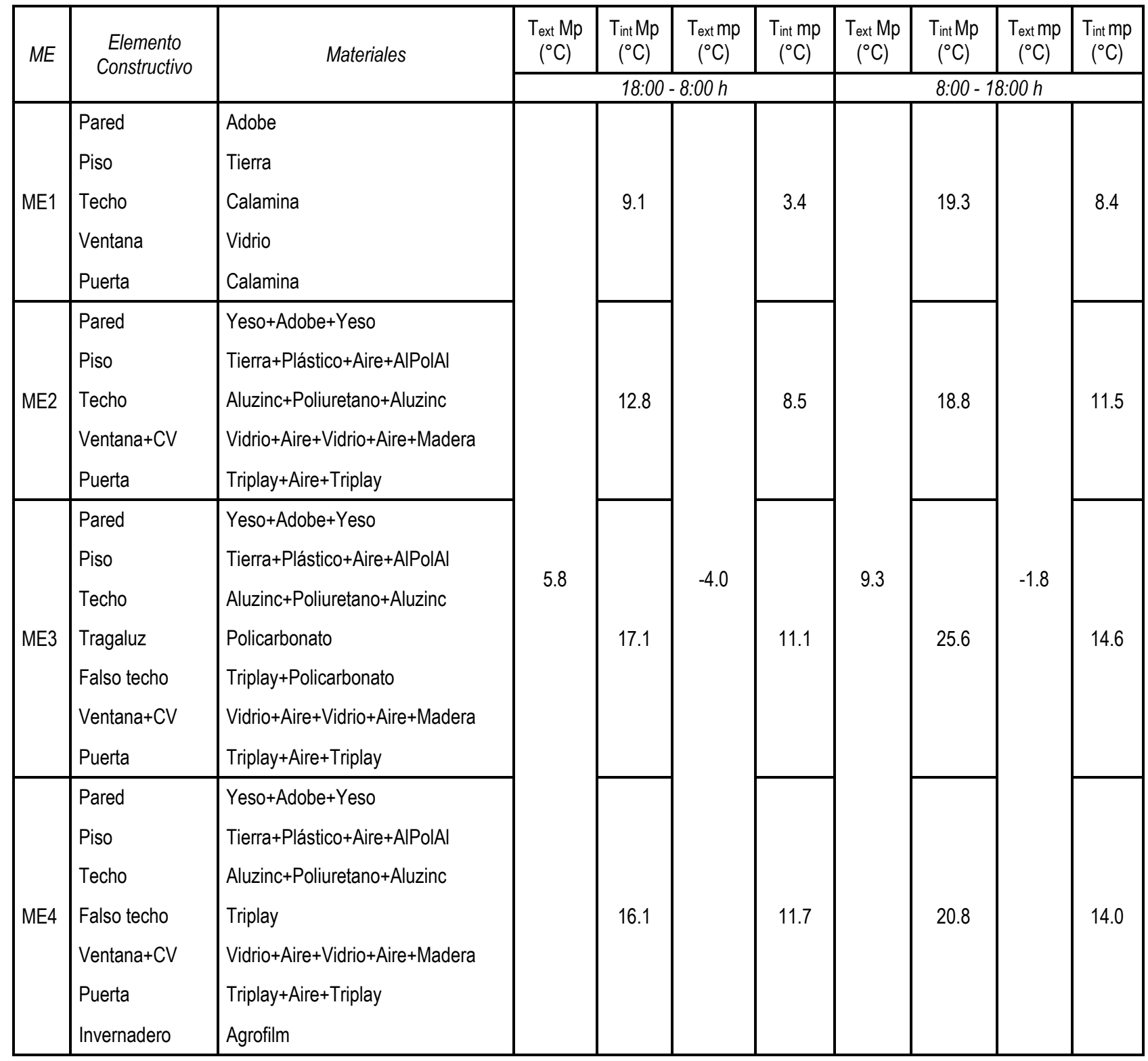


Tabla 2 (continuación)

\begin{tabular}{|c|c|c|c|c|c|c|c|c|c|c|}
\hline \multirow[t]{2}{*}{$M E$} & \multirow{2}{*}{$\begin{array}{l}\text { Elemento } \\
\text { Constructivo }\end{array}$} & \multirow[t]{2}{*}{ Materiales } & $\begin{array}{c}\text { Text Mp } \\
\left({ }^{\circ} \mathrm{C}\right)\end{array}$ & $\begin{array}{l}\text { Tint } \mathrm{Mp} \\
\left({ }^{\circ} \mathrm{C}\right)\end{array}$ & $\begin{array}{c}\text { Text mp } \\
\left({ }^{\circ} \mathrm{C}\right)\end{array}$ & $\begin{array}{c}\mathrm{T}_{\text {int }} \mathrm{mp} \\
\left({ }^{\circ} \mathrm{C}\right)\end{array}$ & $\begin{array}{c}\mathrm{T}_{\mathrm{ext}} \mathrm{Mp} \\
\left({ }^{\circ} \mathrm{C}\right)\end{array}$ & $\begin{array}{l}\text { Tint } \mathrm{Mp} \\
\left({ }^{\circ} \mathrm{C}\right)\end{array}$ & $\begin{array}{c}\text { Text mp } \\
\left({ }^{\circ} \mathrm{C}\right)\end{array}$ & $\begin{array}{c}\text { Tint } \mathrm{mp} \\
\left({ }^{\circ} \mathrm{C}\right)\end{array}$ \\
\hline & & & \multicolumn{4}{|c|}{$18: 00-8: 00 h$} & \multicolumn{4}{|c|}{$8: 00-18: 00 h$} \\
\hline \multirow{8}{*}{ ME5 } & Pared & Yeso+Adobe+Yeso & \multirow{8}{*}{5.8} & \multirow{8}{*}{20.3} & \multirow{8}{*}{-4.0} & \multirow{8}{*}{14.4} & \multirow{8}{*}{9.3} & \multirow{8}{*}{28.1} & \multirow{8}{*}{-1.8} & \multirow{8}{*}{17.3} \\
\hline & Piso & Tierra+Plástico+Aire+AIPolAI & & & & & & & & \\
\hline & Techo & Aluzinc+Poliuretano+Aluzinc & & & & & & & & \\
\hline & Tragaluz+CV & Policarbonato+Madera & & & & & & & & \\
\hline & Falso techo & Triplay+Policarbonato & & & & & & & & \\
\hline & Ventana+CV & Vidrio+Aire+Vidrio+Aire+Madera & & & & & & & & \\
\hline & Puerta & Triplay+Aire+Triplay & & & & & & & & \\
\hline & Invernadero & Agrofilm & & & & & & & & \\
\hline
\end{tabular}

De acuerdo con los resultados de la Tabla 2 proveniente de las simulaciones dinámicas considerando como periodo de ejecución el mes de julio se tiene que, para el análisis de la temperatura interior mínima diaria promedio mensual $\left(\mathrm{T}_{\text {int }} \mathrm{mp}\right.$ ) de 18:00 a 8:00 $\mathrm{h}$ donde se presentan las bajas temperaturas, la contribución debido al aislamiento de la envolvente de ME2, con respecto a ME1, es de $5.1^{\circ} \mathrm{C}$. Respecto de ME2, cuando se agregan tragaluces en el techo y el falso techo (ME3), contribuye a la ganancia de energía que se refleja en un incremento de temperatura de $2.6^{\circ} \mathrm{C}$; al agregar el falso techo y el invernadero adosado a la pared norte (ME4), se obtiene un aumento de energía térmica que representa un incremento de la temperatura de $3.2{ }^{\circ} \mathrm{C}$. $\mathrm{Y}$, al agregar tragaluces en el techo y falso techo con cubiertas de madera (como elementos protectores térmicos), y el invernadero adosado (ME5), se alcanzaría un incremento de $5.9^{\circ} \mathrm{C}$, que además, respecto del mínimo promedio mensual exterior $\left(\mathrm{T}_{\text {ext }} \mathrm{mp}\right.$ ) el incremento sería de $18.4^{\circ} \mathrm{C}$, y $11^{\circ} \mathrm{C}$ respecto de ME1. La Figura 9 muestra el comportamiento promedio horario para el mes de julio (de "heladas") de la temperatura interior de los ME y la temperatura exterior. Entre las 18:00 a 8:00 h, el ME1 y ME5 difieren en promedio en más de $10^{\circ} \mathrm{C}$, y más de $16^{\circ} \mathrm{C}$ entre el ME5 y el exterior. La temperatura mínima se alcanza entre las 6:00 a 7:00 h.

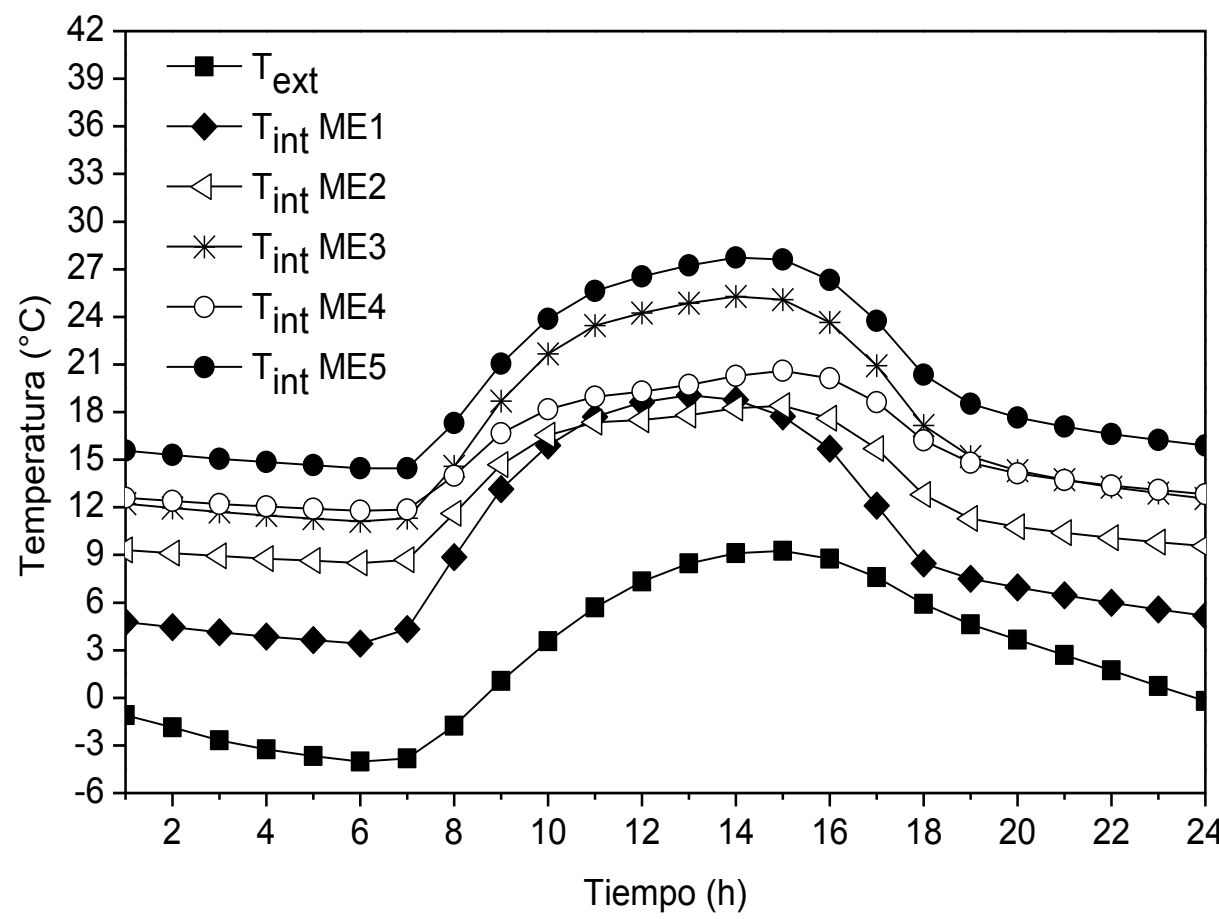

Fig. 9: Comportamiento de la temperatura exterior ( $\left.T_{\text {ext }}\right)$ e interior ( $\left.T_{\text {int }}\right)$ para el mes de julio de los módulo experimentales (ME). 
La simulación del rendimiento térmico del módulo experimental ME5 muestra que se puede alcanzar una temperatura máxima de $28.1^{\circ} \mathrm{C}$ al mediodía, lo que podría generar molestias en las personas pero, dado que generalmente a estas horas las personas están fuera de sus hogares, cuidando su ganado o trabajando en el campo, probablemente esto no sería un problema. Sin embargo, durante ese período del día la energía se almacenaría en las paredes, aprovechando su alta inercia térmica. Por lo tanto, cuando la familia regresa a sus hogares, el ambiente está en un rango de temperatura que les proporciona bienestar térmico. La Figura 10 ilustra la demanda de energía mensual para calefacción de los ME para mantener constante una temperatura interior de bienestar térmico de $16.3^{\circ} \mathrm{C}$ (Molina et al., 2019), donde, anualmente resulta para el caso de ME1, una demanda de energía de $4383.6 \mathrm{kWh}, 1341.3 \mathrm{kWh}$ para ME2, $856.6 \mathrm{kWh}$ para ME3, 667 $\mathrm{kWh}$ para ME4, y $350 \mathrm{kWh}$ para ME5. Y, en ese mismo orden de los ME, se tendría en promedio anual una demanda diaria de $12 \mathrm{kWh}, 3.7 \mathrm{kWh}, 2.3 \mathrm{kWh}, 1.8 \mathrm{kWh}$, y $1 \mathrm{kWh}$, con ahorros energéticos respecto de ME1 de $69 \%$ para ME2, $80 \%$ para ME3, $85 \%$ para ME4, y $92 \%$ para ME5.

Si el rango de bienestar térmico se considera entre 21 y $26^{\circ} \mathrm{C}$ con una temperatura de diseño estandarizada de $22^{\circ} \mathrm{C}$ según la ASHRAE, el requerimiento de energía promedio anual diario para calefacción seria de 21.9 kWh para ME1, 8.1 kWh para ME2, $5.9 \mathrm{kWh}$ para ME3, $5.5 \mathrm{kWh}$ para ME4, y $3.8 \mathrm{kWh}$ para ME5. Si bien la temperatura de diseño de $16.3{ }^{\circ} \mathrm{C}$ para Imata, determinado según modelo adaptativo, conlleva a ahorros energéticos respecto a una temperatura de $22{ }^{\circ} \mathrm{C}(45 \%$ para ME1, $55 \%$ para ME2, $60 \%$ para ME3, y $75 \%$ para ME5), este valor aún es referencial pero puede inferirse que es más adecuado que un valor obtenido para una temperatura estandarizada ya que las personas por sus costumbres, fisiología y psicología, se han adaptado a temperaturas muy bajas. De trabajos previos (Molina et al., 2019), se estima que la zona de bienestar térmico para la población de Imata, según el método de adaptación, puede encontrarse en un mínimo de $12.4^{\circ} \mathrm{C}$ (para el mes de julio) y un máximo de $19.8^{\circ} \mathrm{C}$ (para el mes de diciembre).

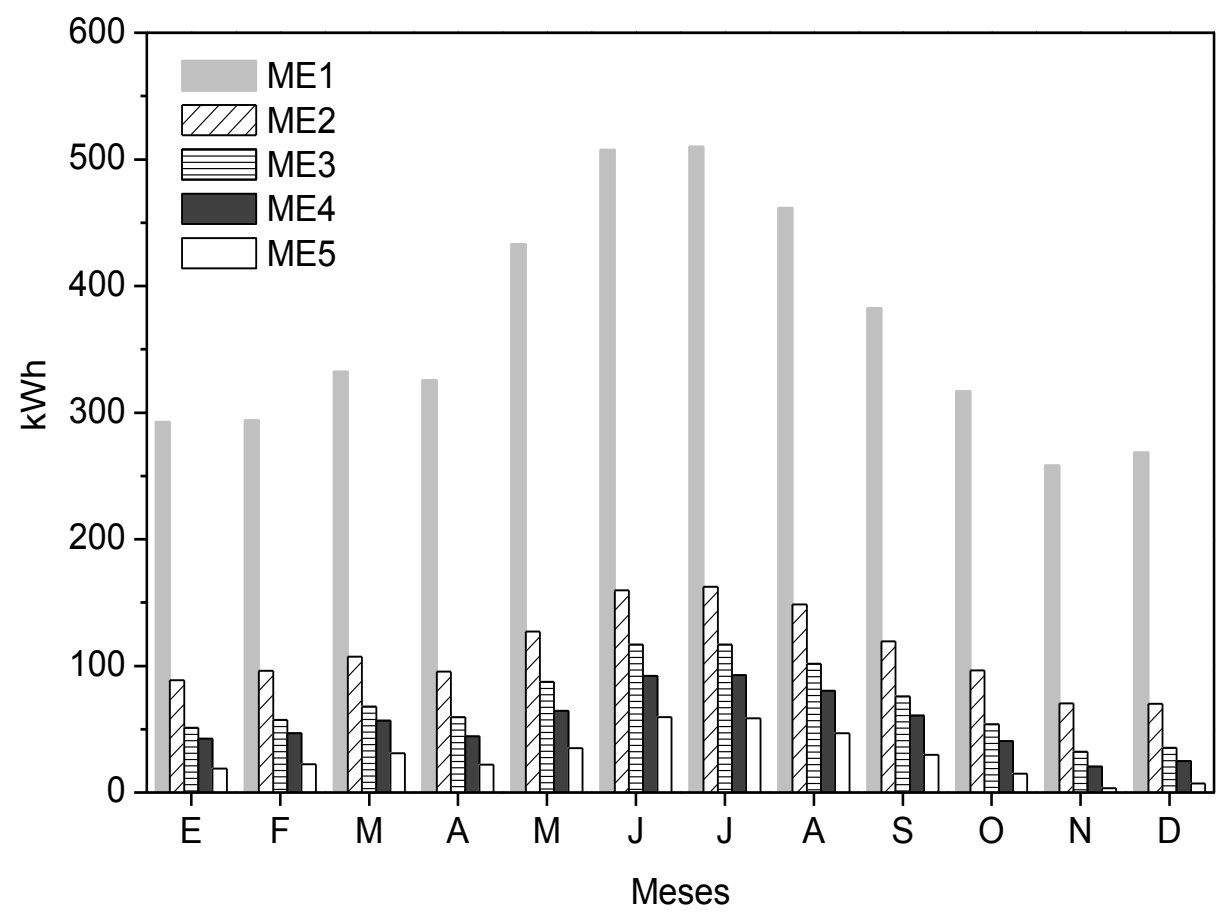

Fig. 10: Demanda mensual de energía requerida para los módulos experimentales (ME) para mantener constante una temperatura interior de bienestar térmico de $16.3^{\circ} \mathrm{C}$.

\section{Análisis de las infiltraciones para el módulo bioclimático ME5}

El análisis del comportamiento térmico dentro de un entorno debe incluir infiltraciones, expresadas como la cuantificación de "Cambios de Aire por Hora", (ACH de los términos en inglés Air Changes per Hour). En la Tabla 3 se muestra los datos de $\mathrm{ACH}$ como los resultados de las simulaciones para el mes de julio divididos en tres rangos de tiempo durante un día: entre las $18: 00 \mathrm{~h}$ a las $6: 00 \mathrm{~h}$ (de $1 \mathrm{a} 8 \mathrm{ACH}$ ) y entre las 6:00 $\mathrm{h}$ y las $8: 00 \mathrm{~h}$ (para 1,4 y $8 \mathrm{ACH}$ ) y entre las $8: 00 \mathrm{~h}$ y las $18: 00 \mathrm{~h}(1 \mathrm{ACH})$. Para los valores de $\mathrm{ACH}$ 1: 1: 1 (el primero y el segundo dígito 1 indican los $\mathrm{ACH}$ entre el exterior y el interior, y el último dígito 1 representa el $\mathrm{ACH}$ entre las zonas térmicas), la temperatura interior mínima promedio disminuye $2.2^{\circ} \mathrm{C}$, mientras que para un $\mathrm{ACH} 8$ : 8: 1 , la temperatura disminuye $9.0^{\circ} \mathrm{C}$ (ver Tabla 3). El comportamiento de estas temperaturas se muestra en la Figura 11, donde se ilustra para el día 15 del mes de julio (día más frío). 
Tabla 3: Comportamiento de las temperaturas exterior $\left(T_{\text {ext }}\right)$ e interior $\left(T_{\text {int }}\right)$ (en $\left.{ }^{\circ} \mathrm{C}\right)$ durante el mes de julio para la construcción bioclimática óptima (ME5) evaluadas para diferentes Cambios de Aire por Hora (ACH).

\begin{tabular}{|c|c|c|c|c|c|c|c|c|c|c|c|}
\hline \multirow{3}{*}{$M E$} & \multicolumn{3}{|c|}{$A C H$} & \multirow{2}{*}{$\begin{array}{c}\mathrm{T}_{\text {ext }} \mathrm{Mp} \\
\left({ }^{\circ} \mathrm{C}\right)\end{array}$} & \multirow{2}{*}{$\begin{array}{c}\mathrm{T}_{\text {int }} \mathrm{Mp} \\
\left({ }^{\circ} \mathrm{C}\right)\end{array}$} & \multirow{2}{*}{$\begin{array}{c}\mathrm{T}_{\text {ext }} \mathrm{mp} \\
\left({ }^{\circ} \mathrm{C}\right)\end{array}$} & \multirow{2}{*}{$\begin{array}{c}\mathrm{T}_{\text {int }} \mathrm{mp} \\
\left({ }^{\circ} \mathrm{C}\right)\end{array}$} & \multirow{2}{*}{$\begin{array}{c}\mathrm{T}_{\text {ext }} \mathrm{Mp} \\
\left({ }^{\circ} \mathrm{C}\right)\end{array}$} & \multirow{2}{*}{$\begin{array}{c}\mathrm{T}_{\text {int }} \mathrm{Mp} \\
\left({ }^{\circ} \mathrm{C}\right)\end{array}$} & \multirow{2}{*}{$\begin{array}{c}T_{\text {ext }} \mathrm{mp} \\
\left({ }^{\circ} \mathrm{C}\right)\end{array}$} & \multirow{2}{*}{$\begin{array}{c}\mathrm{T}_{\text {int }} \mathrm{mp} \\
\left({ }^{\circ} \mathrm{C}\right)\end{array}$} \\
\hline & \multicolumn{2}{|c|}{$18: 00-8: 00 h$} & \multirow{2}{*}{$8: 00-18: 00 h$} & & & & & & & & \\
\hline & $\begin{array}{l}\text { 18:00- } \\
6: 00 \mathrm{~h}\end{array}$ & $\begin{array}{c}6: 00- \\
8: 00 \mathrm{~h}\end{array}$ & & \multicolumn{4}{|c|}{$18: 00-8: 00 h$} & \multicolumn{4}{|c|}{$8: 00-18: 00 h$} \\
\hline \multirow{15}{*}{ ME5 } & 1 & 1 & 1 & \multirow{15}{*}{5.8} & 18.5 & \multirow{15}{*}{-4.0} & 12.2 & \multirow{15}{*}{9.3} & 27.1 & \multirow{15}{*}{-1.8} & 15.0 \\
\hline & 2 & 1 & 1 & & 18.2 & & 10.7 & & 26.8 & & 14.5 \\
\hline & 4 & 1 & 1 & & 17.7 & & 8.5 & & 26.3 & & 13.7 \\
\hline & 6 & 1 & 1 & & 17.3 & & 6.8 & & 25.9 & & 13.2 \\
\hline & 8 & 1 & 1 & & 17.0 & & 5.6 & & 25.6 & & 12.7 \\
\hline & 1 & 4 & 1 & & 18.3 & & 9.8 & & 26.9 & & 12.0 \\
\hline & 2 & 4 & 1 & & 18.0 & & 9.3 & & 26.6 & & 11.6 \\
\hline & 4 & 4 & 1 & & 17.5 & & 8.3 & & 26.1 & & 11.0 \\
\hline & 6 & 4 & 1 & & 17.1 & & 6.7 & & 25.7 & & 10.5 \\
\hline & 8 & 4 & 1 & & 16.9 & & 5.5 & & 25.4 & & 10.1 \\
\hline & 1 & 8 & 1 & & 18.1 & & 7.6 & & 26.7 & & 9.5 \\
\hline & 2 & 8 & 1 & & 17.8 & & 7.2 & & 26.4 & & 9.2 \\
\hline & 4 & 8 & 1 & & 17.3 & & 6.4 & & 25.9 & & 8.7 \\
\hline & 6 & 8 & 1 & & 17.0 & & 5.9 & & 25.5 & & 8.3 \\
\hline & 8 & 8 & 1 & & 16.7 & & 5.4 & & 25.2 & & 8.0 \\
\hline
\end{tabular}

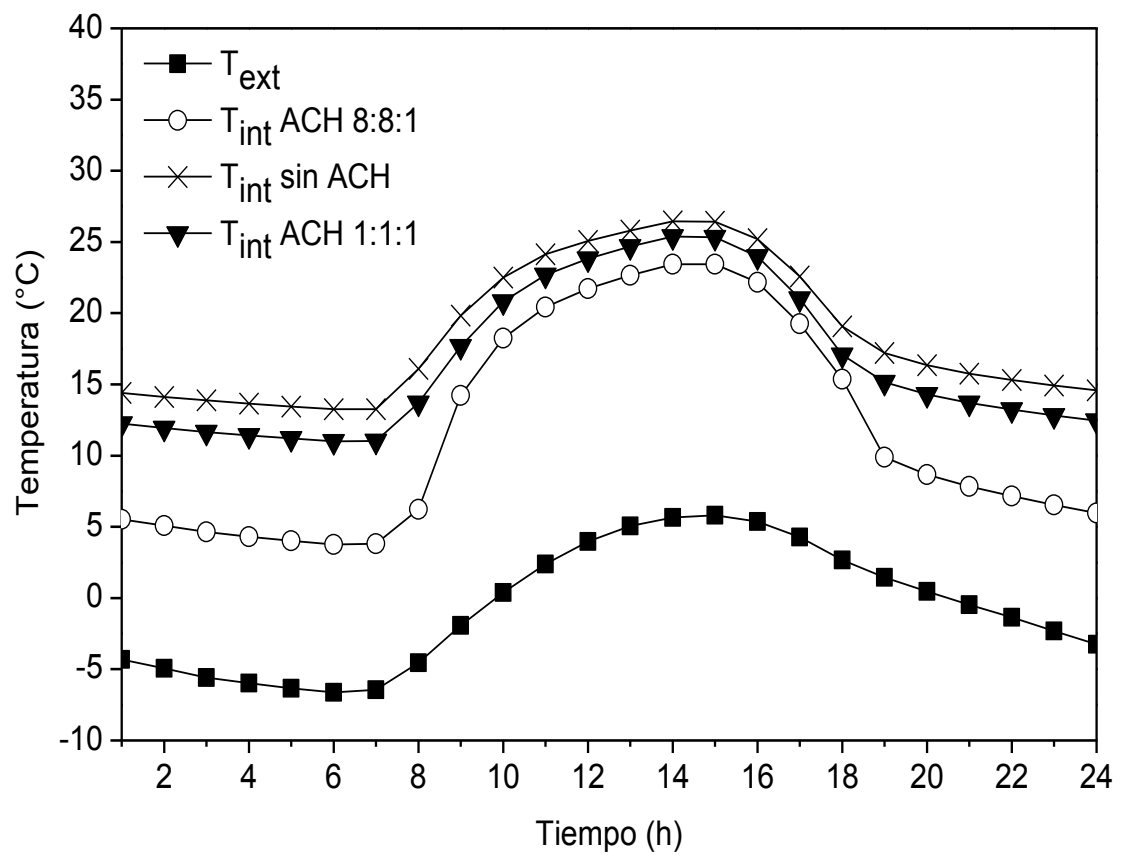

Fig. 11: Comportamiento de la temperatura exterior $\left(T_{\text {ext }}\right)$ e interior $\left(T_{\text {int }}\right)$ para el día más frío de julio para la construcción experimental (ME5) de mejor diseño evaluada para dos diferentes cambios de aire por hora.

La importancia de cuantificar y controlar los $\mathrm{ACH}$ se destaca claramente. Así, para el módulo experimental ME5, se encontró que su rendimiento térmico disminuirá notablemente para ACH mayor a 1: 4: 1 durante las 6:00 y las 8:00 h, período en el que las puertas y ventanas comenzarán a abrirse al comienzo del día, y la temperatura exterior puede ser de $-4{ }^{\circ} \mathrm{C}$. Para el caso de ME5 y $\mathrm{ACH}$ 1: 1: 1 utilizando la estrategia de calentamiento solar pasivo, sería posible estar dentro de la zona de bienestar térmico. 


\section{CONCLUSIONES}

Los resultados y análisis de las simulaciones dinámicas muestran que es posible mediante el empleo de diferentes técnicas constructivas de aislamiento térmico, masa térmica, e inclusión de sistemas de climatización natural como los tragaluces e invernadero adosado al ME, como el caso del ME5 respecto de ME1, se pueden obtener incrementos de temperaturas interiores mínimas promedios para el mes de $11^{\circ} \mathrm{C}, \mathrm{y}$ $18.4^{\circ} \mathrm{C}$, respecto a la temperatura mínima promedio exterior. Con un ahorro de energía diario del $92 \%$ para ME5 (1 kWh) respecto de ME1 $(12 \mathrm{kWh})$, para mantener una temperatura interior constante de $16.3^{\circ} \mathrm{C} . \mathrm{Y}$ demás ahorros energéticos respecto de ME1 de $69 \%$ para ME2, $80 \%$ para ME3, y $85 \%$ para ME4.

Dentro del contexto de alcanzar temperaturas interiores de bienestar térmico según modelo adaptativo, tanto en los ME como en todo tipo de edificio o vivienda, la hermeticidad juega un rol importante que de no presentarse, las infiltraciones de aire $(\mathrm{ACH})$ van a contribuir a una reducción de la temperatura interior de hasta en 50\%, tal como resultó para el ME5 donde, un $\mathrm{ACH}$ de 8 entre las 18:00 y 6:00 h, y de 6:00 a 8:00 h, con un $\mathrm{ACH}$ de 1 entre las 8:00 y $18: 00 \mathrm{~h}$, puede reducir la temperatura interior de $15^{\circ} \mathrm{C}$ a $8{ }^{\circ} \mathrm{C}$ respecto de un $\mathrm{ACH}$ de 1 para las mismas horas. Finalmente, los resultados obtenidos en este trabajo permitirán una construcción adecuada del ME que deberá ser validada experimentalmente.

\section{AGRADECIMIENTOS}

Este trabajo se ha realizado por la financiación recibida por el Consejo Nacional de Ciencia, Tecnología e Innovación (CONCYTEC) a través del convenio 024-2018-FONDECYT. J.M agradece la beca de doctorado recibida por medio del contrato 05-2018 FONDECYT/BM.

\section{NOTACIÓN}

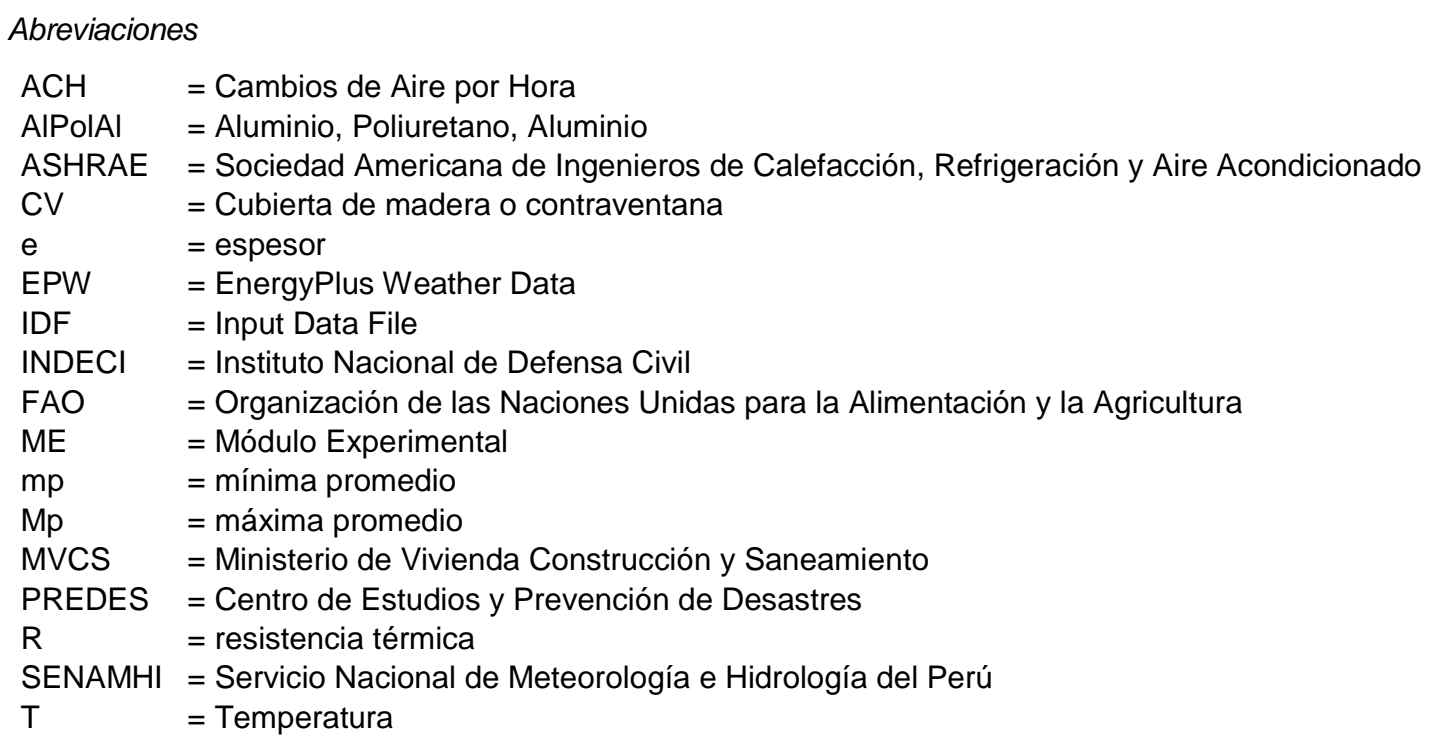

Súper/sub índices

\begin{tabular}{|c|c|}
\hline ext & $=$ exterior \\
\hline int & $=$ interior \\
\hline Maxp & = máxima promedio \\
\hline med & $=$ media \\
\hline mext & = mínima extrema \\
\hline Mext & = máxima extrema \\
\hline $\operatorname{minp}$ & = mínima promedio \\
\hline sup Int & $=$ superficie interior \\
\hline p Ext & $=$ superficie exterior \\
\hline
\end{tabular}

\section{REFERENCIAS}

Abanto, G.A., Karkri M. y otros cuatro autores, Thermal Properties of Adobe employed in Peruvian Rural Areas: Experimental Results and Numerical Simulation of a Traditional Bio-Composite Material, https://doi.org/10.1016/j.cscm.2017.02.001, Case Stud. Constr. Mater., 6, 177-191 (2017).

Agugliaro, F.M., Montoya F.G., Ortega A.S. y Cruz A.G., Review of Bioclimatic Architecture Strategies for Achieving Thermal Comfort, https://doi.org/10.1016/j.rser.2015.04.095, Renew. Sust. Energ. Rev., 49, 736-755 (2015). 
Albatayneh, A., Alterman D., Page A. y Moghtaderi B., Temperature versus energy based approaches in the thermal assessment of buildings, https://doi.org/10.1016/j.egypro.2017.09.013, Energy Procedia, International Scientific Conference "Environmental and Climate Technologies", CONECT 2017, 10-12 May 2017, Riga, Latvia 128, 46-50 (2017).

Alghoul, S.K., Rijabo H.G. y Mashena M.E., Energy Consumption in Buildings: A Correlation for the Influence of Window to Wall Ratio and Window Orientation in Tripoli, Libya, https://doi.org/10.1016/j.jobe.2017.04.003, J. Build. Eng., 11, 8286 (2017).

ASHRAE, ANSI/ASHRAE Standard 55-1992: Thermal Environmental Conditions for Human Occupancy, ISSN: 1041-2336, American Society of Heating, Refrigerating and Air-Conditioning Engineers, Inc. (ASHRAE) (1992).

Calderón, R., Arredondo J.A., Gallegos R. y Mayagoitia F., Reducción del Consumo Eléctrico y CO(2) mediante Sistemas de Ahorro y de Aislamiento Térmico aplicados a Viviendas en Zonas Áridas de México, http://dx.doi.org/10.4067/S071807642011000200008, Inf. Tecnol. 22, 69-78 (2011).

Crawley, D.B., Lawrie L.K. y otros cinco autores, EnergyPlus: Creating a New-Generation Building Energy Simulation Program, https://doi.org/10.1016/S0378-7788(00)00114-6, Energy Build., 33(4), 319-331 (2001).

Flores, A., Construcción de una Vivienda Solar en Base a las Propiedades Termofísicas y Evaluación Experimental de su Confort Térmico en llave, http://dx.doi.org/10.18271/ria.2014.101, Journal of High Andean Research, 16(01) (2014).

Fumo, N., Mago P. y Luck R., Methodology to Estimate Building Energy Consumption using EnergyPlus Benchmark Models, https://doi.org/10.1016/j.enbuild.2010.07.027, Energy Build., 42(12), 2331-2337 (2010).

Givoni, B., Comfort, Climate Analysis and Building Design Guidelines, https://doi.org/10.1016/0378-7788(92)90047-K, Energy Build., 18(1), 11-23 (1992).

Gómez, M.M., Espinoza R. y Horn M., Energy for Unserved Populations, In: IANAS, editor. Guide towards a Sustainable Energy Future for the Americas, ISBN: 978-607-8379-25-5, IANAS-IAP, México, pp. 38-61 (2016).

Harish, V.SKV. y Kumar A., A Review on Modeling and Simulation of Building Energy Systems, https://doi.org/10.1016/j.rser.2015.12.040, Renew. Sust. Energ. Rev., 56, 1272-1292 (2016).

Lefebvre, G., Caractérisation de l'inertie Thermique d'un Bâtiment par Analyse Modale, Revue générale de thermique, 28 (332-333), 501-512 (1989).

Madrigal, J.A., Cabello J.J., Sagastume A. y Balbis M., Evaluación de la Climatización en Locales Comerciales, Integrando Técnicas de Termografía, Simulación y Modelado por Elementos Finitos, http://dx.doi.org/10.4067/S071807642018000400179, Inf. Tecnol. 29, 179-188 (2018).

Molina, J.O., Espinoza R.L., Horn M.J. y Gómez, M.M., Thermal Performance Evaluation of Isolation and two Active Solar Heating Systems for an Experimental Module: A Rural Peruvian case at 3700 masl, https://doi.org/10.1088/17426596/1173/1/012003, J. Phys.: Conference Series, 1173, 012003 (2019).

Morillón, D., Atlas del Bioclima de México, ISBN: 970-32-1904-7, Instituto de Ingeniería, UNAM, México, D.F. (2004).

MVCS, Norma EM.110 Confort Térmico y Lumínico con Eficiencia Energética, Reglamento Nacional de Edificaciones, Lima - Perú (2014).

Nicol, J.F. y Humphreys M.A., Adaptive Thermal Comfort and Sustainable Thermal Standards for Buildings, https://doi.org/10.1016/S0378-7788(02)00006-3, Energy Build, 34(6), 563-572 (2002).

Peel, M.C., Finlayson B.L. y McMahon T.A., Updated world map of the Köppen-Geiger climate classification, https://doi.org/10.5194/hess-11-1633-2007, Hydrol. Earth Syst. Sci., 11, 1633-1644 (2007).

PREDES, Mapa de Heladas (https://www.predes.org.pe/mapas/heladas/, acceso: 29/11/2019), Lima -Perú (2016)

SENAMHI-FAO, Atlas de Heladas del Perú: Convenio Interinstitucional Senamhi-FAO, Lima- Perú (2010).

Silvero, F., Rodrigues F. y otros tres autores, The Path Towards Buildings Energy Efficiency in South American Countries, https://doi.org/10.1016/j.scs.2018.10.028, Sustainable Cities and Society, 44, 646-665 (2019).

Tsoka, S., Tolika K., Theodosiou T. y Tsikaloudaki K., Evaluation of Stochastically Generated Weather Datasets for Building Energy Simulation, https://doi.org/10.1016/j.egypro.2017.07.449, Energy Procedia, 122, $853-858$ (2017).

Velazco, B.N., García J.R. y Matsumoto Y., Analysis and Evaluation of Bioclimatic Systems to Characterize their Performance in Experimental Modules, https://doi.org/10.1016/j.egypro.2017.07.439, Energy Procedia, 122, 1093-1098 (2017).

Verbeke, S. y Audenaert A., Thermal Inertia in Building: A Review of Impacts Across Climate and Building Use, https://doi.org/10.1016/j.rser.2017.08.083, Renew. Sust. Energ. Rev., 82, 2300-2318 (2018).

World Bank Group, Global Solar Atlas 2.0 (https://solargis.com/maps-and-gis-data/download/peru, acceso: 29/9/(2019) 
